\title{
AWLA y Eduwebman: Una propuesta para el desarrollo de Sistemas de e-learning colaborativos en web.
}

\author{
Manuel ortega \\ Manuel.ortega@uclm.es \\ Pedro $P$. Sánchez Villalón \\ ppsv@telefonica.net \\ Carlos Peces \\ cpeces@varnet.com \\ Universidad de Castilla La Mancha
}

\section{Introducción}

Asistimos al despegue de una generación de sistemas para la gestión de cursos en Web que ha producido más de una centena de "Sistemas Gestores de Aprendizaje" tanto de pago como gratuitos o con licencia "Open Source".

Según el Glosario sobre e-Learning del Departamento del Tesoro del Gobierno de los Estados Unidos un "Learning Management System” (en castellano, Sistema de Gestión del Aprendizaje, más conocido por las siglas del acrónimo en inglés LMS) puede definirse de la siguiente manera (IRS, 2004):

- Aplicación software o tecnología basada en Web usada para planificar, implementar y evaluar un proceso de aprendizaje específico. Típicamente, un LMS provee al instructor de una forma para crear y distribuir contenidos, monitorizar la participación de los estudiantes, y evaluar el aprendizaje del alumno. Un LMS puede proporcionar al alumno características interactivas como foros de distintos temas, videoconferencia, etc. El Advanced Distance Learning Group, del Departamento de Defensa de los Estados Unidos ha creado un conjunto de especificaciones llamado Shareable Content Object Reference Model (SCORM) para facilitar la estandarización de los LMS.

- Por otra parte empieza a hablarse de un nuevo concepto de LMS en el que los contenidos adquieren más importancia: Los LCMS (Learning Content Management System) añaden a los anteriores las posibilidades de creación de contenidos además de otras características.

En la definición anterior aparece una de las líneas de mejora más importante de los Sistemas Gestores de Aprendizaje: la utilización de estándares de desarrollo de contenidos que permitan el intercambio de objetos de aprendizaje. Un “Objeto de Aprendizaje” (Learning Object) (IRS, 2004) es "una porción de información reutilizable, independiente del medio, usado como un bloque modular para la construcción de contenidos de e-learning. Los objetos de aprendizaje son más efectivos cuando se organizan mediante un sistema de clasificación de metadatos y almacenados en un repositorio de datos como, por ejemplo un LCMS

El número de LMS actuales se ha disparado y un administrador de sistemas de aprendizaje debe conocer las principales características de los sistemas comerciales y de los que soportan código libre. El número de Universidades que optan a sistemas mixtos de aprendizaje (Blended Learning, en inglés) en los que se combinan el estudio en línea junto al aprendizaje presencial hace necesario un período de reflexión que no pocas veces se ha eliminado en aras de la rapidez en la instalación de los primeros gestores de cursos. El resultado obtenido puede imaginarse: la precipitación en la elección de sistemas ha llevado a la búsqueda errante del mejor LMS entre los más caros, que no siempre son los más eficientes. Pero esta discusión no forma parte de este artículo. 
En las siguientes secciones veremos, en primer lugar un repaso a los principales gestores de aprendizaje desarrollados hasta ahora, para posteriormente desarrollar algunas de las propuestas que a juicio de los autores deberían ofrecer los sistemas LMS. Por último presentaremos algunos desarrollos del grupo CHICO [3] , para terminar con unas conclusiones sobre las propuestas presentadas en este artículo.

\section{Los Sistemas Gestores de Aprendizaje actuales}

El número de Gestores de Aprendizaje actuales supera posiblemente la centena, aunque de entre éstos solamente menos de una docena tienen una implantación suficiente como para que una institución universitaria opte por ellos. Para tener una idea bastante exacta de las posibilidades que presenta cada uno de ellos puede consultarse la Web de EduTools (EduTools, 2004). En esta Web pueden realizarse comparativas configurables por el usuario sobre más de 40 variables que miden todas las principales características de los sistemas de e-Learning.

La primera clasificación que debemos hacer en los LMS es entre los que tienen un coste en cuanto a su licencia y aquellos que se ofrecen como código libre (Open Source). Entre los sistemas de pago el más conocido es sin duda WebCT[1] ,. En cuanto a los sistemas libres hay una clara competencia entre varios de ellos entre los que cabe citar ATutor, Moodle, Claroline y LRN[2]. Existen además otros sistemas pensados para la colaboración en línea o basados en wiki que a menudo se utilizan con propósitos docentes.

Para poder clasificar las características principales de los LMS actuales debemos concentrarnos al menos en los siguientes aspectos: Herramientas disponibles para los alumnos, herramientas de gestión, es decir, aquellas usadas por los administradores del sistema o por los profesores/autores de los contenidos y por último características técnicas de los sistemas. También podemos constatar una serie de deficiencias observadas de los LMS atendiendo a los factores de calidad de software. Los más significativos son los siguientes: el bajo rendimiento del sistema cuando el número de cursos aumenta, lo que lleva a la frustración del alumno que no ve avanzar su aprendizaje por la lentitud de respuesta del sistema, coste excesivo de actualización y mantenimiento del sistema, modificabilidad y adaptabilidad restringidas, limitadas capacidades de portabilidad y reusabilidad de los recursos de aprendizaje creados y prácticamente ninguna interoperabilidad entre distintos LMS. Además y como aspecto destacado de la evaluación tenemos que hacer notar la falta de usabilidad de los sistemas consultados y la nula accesibilidad de la mayoría de ellos.

Para una revisión en detalle de estos sistemas y de sus principales factores de posible mejora puede consultarse el artículo referenciado en (Ortega, 2004a).

En la actualidad una de las vías para lograr una mayor calidad de los sistemas software se basa en la creación de estándares para la descripción arquitectónica de los sistemas software o para la gestión de objetos de aprendizaje reutilizables. Entre las medidas tomadas caben destacar las del IEEE Learning Technology Standards Committee en su iniciativa Learning Technology Systems Architecture (LTSA) (IEEE, 2001), el estándar europeo a través del proyecto ARIADNE (ARIADNE, 2004), la iniciativa británica CETIS (CETIS, 2001) o la Open Knowledge Initiative (OKI) (Thorne et al., 2002).

Ya empiezan a surgir estudios sobre calidad de software que determinan los parámetros de los LMS en varios apartados: atributos de calidad que pueden discernirse en el tiempo de ejecución y aquellos que no se pueden discernir por la ejecución (Avgeriou et al., 2002) y por último en sus características como negocio. Entre las características del primer grupo se encuentran el rendimiento, la seguridad, el tiempo de trabajo sin que se produzcan cortes en el acceso, la usabilidad, y la confiabilidad del sistema. En cuanto a las características que no se pueden descubrir en la ejecución están la modificabilidad, relacionada con la mantenibilidad, la portabilidad, la interoperabilidad, la reusabilidad y la posibilidad de crear tests sobre el sistema para ver sus resultados. Entre los parámetros relacionados con la faceta comercial de los LMS están el tiempo necesario para salir al mercado, el coste, el tiempo de vida previsto del sistema y el mercado al que se destina el producto.

\section{Necesidades pedagógicas de los LMS}

Si desde la perspectiva de la calidad del software los LMS actuales tienen que evolucionar bastante, las 
características que deben disponer los LMS para que resulten eficaces desde el punto de vista pedagógico son muchas y no parece que en ocasiones se vayan a introducir de manera sencilla en los sistemas creados.

Existe una gran tradición de sistemas utilizados en la Informática Educativa que han ido evolucionando desde los primitivos sistemas CAI (Computer Aided Instruction) a lo largo de más de tres décadas de investigación. Pero como suele suceder en la Ciencia y sobre todo en la Ingeniería, cuando una revolución científica o tecnológica nos provee de nuevas formas de trabajo, olvidamos los estudios precedentes en aras de la utilización del nuevo juguete que la técnica nos ha regalado. Así ocurrió tras el despegue de la Web como paradigma de sistema hipermedia, donde se olvidaron los años de investigación en Hipertexto, y así está sucediendo también en la utilización de la Web para la gestión de contenidos de cursos. Porque éste es el uso real de Internet para el aprendizaje en la actualidad: sólo la gestión de contenidos.

Los sistemas LMS están en este momento sirviendo como contenedores de cursos que pueden ser recibidos, eso sí, casi inmediatamente desde sus autores a sus receptores. Pueden mejorarse estos contenidos casi sin coste, se puede interaccionar con el autor por correo electrónico y se puede colocar algún contenido interactivo con el añadido de "saltar la introducción” tan útil en el 99 \% de los casos. Y poco más. Ésta es la visión de los autores de este artículo y las posibles soluciones vamos a explicarlas en las siguientes líneas.

Para empezar, un LMS o un LCMS no puede ser un libro. Es decir no podemos pensar en estos sistemas como si sólo fueran un conjunto de apuntes para nuestros alumnos. En los LMS deben ser posibles, por ejemplo, no sólo los tests de autoevaluación sino que también debemos presentar problemas para su solución de forma individual o en grupo, sobre todo cuando nos enfrentamos a asignaturas de alto contenido experimental.

Uno de los aspectos en los que tienen que mejorar los LMS es en el tema de los contenidos interactivos. Las páginas estáticas de contenidos pueden ser válidas para determinadas asignaturas o para los aspectos iniciales de presentación del tema, pero sobre todo en las materias experimentales no pueden ser los únicos contenidos. En realidad el problema no es de los LMS sino de sus autores, ya que en cualquier caso los contenidos audiovisuales o las animaciones de tipo Macromedia Flash son fáciles de introducir en una página Web. El problema es saber hacerlos.

Pero la interactividad tiene que ver no sólo con los contenidos multimedia. Requiere la intervención del usuario. Además, el usuario no es un tipo homogéneo de persona, sino que requiere atención especializada. De nuevo tenemos que decir que hemos olvidado en los LMS toda la trayectoria de los sistemas hipermedia adaptativos que se acomodan a las necesidades de aprendizaje del usuario, siguiendo la corriente constructivista del desarrollo del conocimiento que parece subyacer en la aplicación de las tecnologías de la información y las comunicaciones (TIC) al aprendizaje en la actualidad. Un ejemplo de este estilo de adaptatividad es PlanEdit del grupo CHICO (Redondo et al., 2002). PlanEdit a partir de un plan general de diseño propuesto por un experto y la especificación del problema de diseño que realiza el profesor, genera un plan óptimo de diseño adaptado al problema propuesto. Éste, junto al nivel de ayuda que sugiere el profesor dependiendo del alumnado que ha de resolver el problema, define la forma de corrección al alumno de su propuesta y el nivel de ayuda que recibe.

Precisamente el nivel de ayuda que debe tener el alumno al enfrentarse a un problema a resolver es muy importante que se integre en los LMS. En éstos suele aparecer una ayuda sobre cómo utilizar el LMS como alumno. Pero debemos disponer de ayudas en línea para la resolución de problemas de forma individual o en grupo. Cuando el alumno empieza a resolver los primeros problemas, éstos son sencillos y el nivel de ayuda por el contrario debe ser alto. Cuando el alumno ha progresado en su conocimiento los problemas a resolver son más difíciles y el grado de ayuda es o muy escaso o nulo. A esto se le denomina andamiaje (scaffolding) y es una de las técnicas aplicadas en los sistemas modernos de enseñanzaaprendizaje. Domosim-TPC también dispone de un sistema de andamiaje.

También se han olvidado en la creación de cursos en LMS las lecciones aprendidas en los Sistemas Tutores Inteligentes (ITS, Intelligent Tutoring Systems). Con respecto a este aspecto también podemos citar diversos desarrollos de CHICO sobre Domosim y Domosim-TPC (Bravo et al., 2002; Bravo et al., 2003). Las características que convierten un sistema de enseñanza - aprendizaje en un Sistema Tutor 
Inteligente son muchas, pero pueden resumirse en la necesidad de tener un modelo del alumno, un módulo del dominio a estudiar y un módulo del profesor que, de forma coordinada, tutorizan las acciones del alumno en su trabajo.

Otro tema que no está bien resuelto en los LMS es la posibilidad de realizar Simulaciones en las que los alumnos tengan que descubrir las leyes que rigen un determinado proceso. Esto se llama Aprendizaje por Descubrimiento y es la base de los sistemas desarrollados por CHICO (Redondo et al, 2003a; Bravo et al., 2005). Se ha demostrado la enorme utilidad del aprendizaje por descubrimiento ya que lo que se averigua por uno mismo se retiene en la memoria mucho más que lo que se memoriza.

Otra gran deficiencia de los sistemas LMS actuales es que los aspectos de trabajo colaborativo utilizados son muy limitados. Muchos sistemas gestores de aprendizaje utilizan los foros y el correo electrónico (tanto interno como externo) para llevar a cabo la colaboración. Los mecanismos de conciencia de grupo (awareness, en inglés) son casi inexistentes y la colaboración sincrónica sólo se lleva a cabo en muy pocos sistemas mediante pizarras compartidas muy sencillas, que no aportan nada a las que ya se pueden utilizar en aplicaciones como Microsoft Messenger. En nuestro sistema Domosim-TPC hemos tenido en cuenta tanto la colaboración asincrónica como la sincrónica de distintas maneras y para distintos espacios de trabajo, usando también diferentes técnicas de awareness (Bravo et al., 2002; Redondo et al, 2003a; Bravo et al., 2005).

En estos sistemas colaborativos donde hay que resolver primero de forma individual un problema y después con la ayuda de otros refinar la solución son muy importantes los sistemas de evaluación de la solución individual, de la solución colectiva, del proceso de discusión y argumentación y de las consecuencias de este proceso sobre la solución propuesta. Éste es otro problema del que adolecen los sistemas LMS que el grupo CHICO ha intentado remediar en Domosim -TPC (Redondo et al., 2003b).

Como última contribución a los sistemas LMS, CHICO piensa que éstos deben contar con la posibilidad de trabajo en cualquier momento y en cualquier lugar. Esta filosofía se basa en la denominada Computación Ubicua, cuyo inspirador fue Mark Weiser (1991). CHICO propuso la computación ubicua como paradigma del aula de nuestro tiempo (Ortega, 2000) en 1999. Los sistemas AULA (Paredes et al., 2003), que utiliza PDAs para actividades de aprendizaje del inglés, y AWLA (Sánchez-Villalón et al., 2004; Ortega et al., 2005), servicio para el aprendizaje y práctica de la escritura basado en Web, participan de esta filosofía, la de posibilitar el trabajo del alumno dentro y fuera del aula. En el caso de AULA, basado en PDA, se hace necesaria la sincronización de contenidos cuando se vuelve al aula de trabajo o cuando se tiene la posibilidad de conectarse al sistema. Ni que decir tiene que muy pocos sistemas LMS cuentan con esta posibilidad, que es sin duda una de las características que más se van a demandar en el futuro a los sistemas de apoyo al aprendizaje.

\section{Eduwebman y AWLA: Propuestas de Sistemas E-Learning}

CHICO está desarrollando un LMS para la gestión de actividades de aprendizaje en el entorno de una Escuela de Ingeniería en Informática. El proyecto se denomina Eduwebman y ha comenzado con tres proyectos de fin de carrera que desarrollan la primera versión del sistema, ya en uso, y las versiones extendidas, Eduwebman Pro y ubicua, Eduwebman Mobile, de este sistema (Figura 1). Aunque nos encontramos en su estadio inicial, nos proponemos seguir las guías de diseño del sistema que hemos configurado en este artículo, proponiendo un sistema LMS acorde a las necesidades de nuestro entorno. 


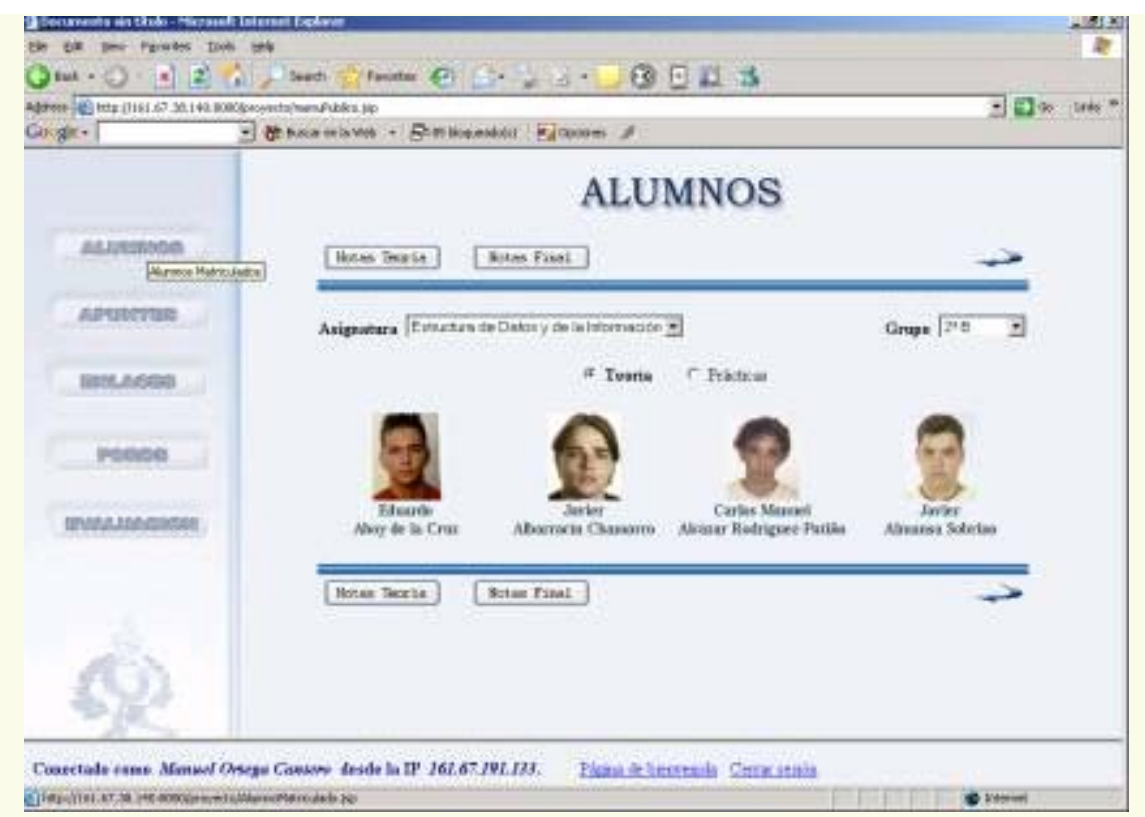

Fig. 1. Entorno LMS Eduwebman.

Por otro lado, el sistema de escritura para el aprendizaje AWLA, basado en Web, intenta dar un paso cualitativo en la provisión de recursos interactivos para el aprendizaje. Siguiendo la tendencia actual de la Web de proporcionar recursos para el aprendizaje más interactivos, AWLA intenta formar parte del diseño de un entorno de aprendizaje más avanzado basado en escenarios, donde la provisión y acceso a contenidos no es tan crucial como el hecho de facilitar el acceso a servicios Web, basados en agentes que ayudan al aprendizaje, bien sean utilidades para la comunicación (principalmente escrita) entre el profesor y el alumno, y entre los alumnos entre sí, como bibliotecas digitales, diccionarios, glosarios, búsquedas Web predefinidas, acceso a materiales y contenidos externos, etc. Estos entornos interactivos permiten diseñar tareas o actividades de aprendizaje, realizar un seguimiento de las acciones del usuario, adaptar el escenario según la evolución del aprendizaje del alumno y ofrecer alternativas adicionales en su proceso de aprendizaje. Los profesores no sólo no son ya transmisores de su conocimiento en cuanto a información se refiere, sino mentores o tutores que facilitan el aprendizaje. Tampoco se convierten, como existe el peligro con los LMS tradicionales, en meros mediadores o selectores de contenidos, que guían a los alumnos hacia la información más apropiada y actualizada, sino que además diseñan las actividades de aprendizaje, que puede estar basado en tareas, en resultados, en simulación o en meras experiencias. Es la experiencia del aprendizaje lo que importa en estos nuevos entornos interactivos. Deben ofrecer recursos de colaboración en grupo y de actividades individualizadas para el aprendizaje desde cualquier lugar y en cualquier momento. Estas actividades de aprendizaje deben estar dirigidas a ayudar al alumno a desarrollar el conocimiento que requiere una disciplina, a la vez que servir de experiencia para el uso de recursos disponibles para el aprendizaje continuo, a lo largo de toda la vida (life-long learning, en inglés).

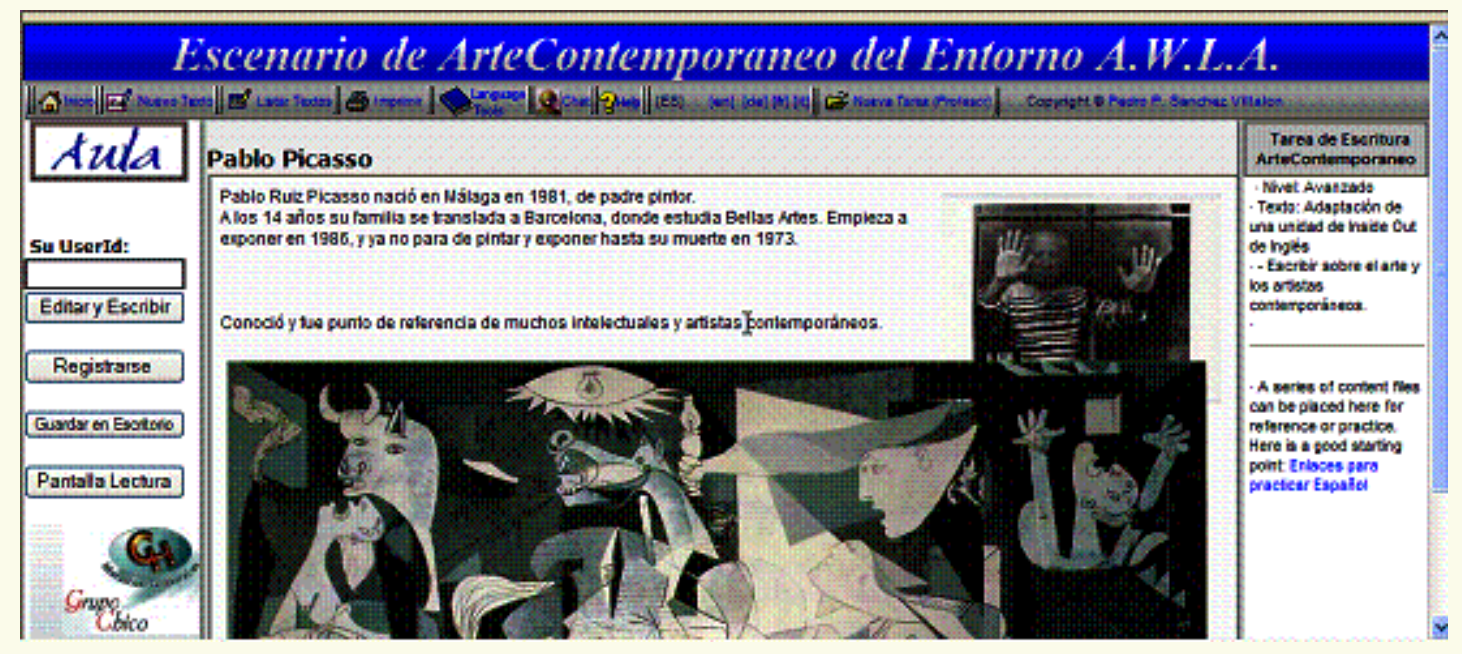

Fig. 2. Pantalla de inicio de escritura de un documento con AWLA (para español) 
AWLA [4] (Figura 2) intenta desarrollar los aspectos ubicuos (en cualquier momento y en cualquier lugar) de la capacidad de escribir en la Web como herramienta de comunicación, integrable en cualquier entorno de aprendizaje. Permite utilizar la escritura como uno de los elementos básicos del desarrollo de conocimiento, con el que el alumno trasmite exteriorizando (publicando) su conocimiento sobre una disciplina en forma escrita tanto para comunicarlo al profesor (que puede hacer un seguimiento del proceso de elaboración del texto) como para compartirlo con los compañeros y, convenientemente al final del proceso, con cualquier usuario que pueda tener acceso a través de la Web a su entorno de aprendizaje. El sistema permite escribir información y presentarla en página Web al instante, sin necesidad de saber editar código HTML a la vez que proporcionando la posibilidad de su edición final en el ordenador de escritorio, portátil, o PDA personal como documento .rtf (de texto con formato enriquecido) o .doc editable con Microsoft Word ${ }^{\circledR}$ y facilitar su presentación impresa.

AWLA también ofrece la capacidad de escritura en forma de diálogo a través de un chat en Web, con el que comunicarse con el profesor o con los compañeros tanto de forma síncrona como asíncrona. Esta utilidad del chat es esencial en la elaboración colaborativa de textos escritos. Otra utilidad que ofrece AWLA al profesor es la de poder diseñar la tarea, presentarla en todo momento a los alumnos y modificarla en cualquier momento durante el desarrollo de la misma dependiendo de la evolución del proceso de aprendizaje en particular. El sistema es adaptable a cualquier disciplina donde la escritura sea herramienta común de comunicación entre los participantes en el proceso de aprendizaje.

Estos nuevos sistemas de aprendizaje e-Learning, en definitiva, permiten utilizar las nuevas herramientas que ofrece la tecnología y las características de ubicuidad y las posibilidades de colaboración que ofrece el nuevo entorno de aprendizaje en Web.

\section{Conclusiones}

Los sistemas LMS actuales no responden a las expectativas que sobre ellos tienen las instituciones encargadas de planificar su uso. No pueden ser simples almacenes de información que se utilizan como un libro de texto.

La utilización de sistemas complejos capaces de crear actividades de aprendizaje que puedan ser tuteladas, con ayudas en línea que aprendan de la evaluación del alumno, el uso de mecanismos propios de la Inteligencia Artificial, basados en agentes de aprendizaje más que sólo en objetos de aprendizaje, los sistemas colaborativos de enseñanza y el paradigma de la Computación Ubicua harán que estos sistemas empiecen a considerarse como verdaderas ayudas en el proceso de enseñanza - aprendizaje al que los informáticos y los pedagogos estamos obligados a contribuir teniendo en cuenta el Espacio Europeo de Educación Superior. El camino hacia el Aprendizaje Mixto, presencial y a distancia (Blended Learning) parece haber comenzado.

\section{Bibliografía}

ARIADNE, (2004) en http://www.ariadne-eu.org.

AVGERIOU, P., RETALIS, S. SKORDALAKIS, E. (2002) Building Quality Into Learning Management Systems - An Architecture-Centric Approach, en International Workshop on Conceptual Modeling Quality (IWCMQ'02), Tampere, Finland.

BRAVO, C., REDONDO, M.A., ORTEGA, M., VERDEJO, F. (2002) Collaborative Discovery Learning of Model Design; "Intelligent Tutoring Systems”, Proceedings of Intelligent Tutoring Systems ITS 2002. LNCS 2363; Springer Verlag; 671-680.

BRAVO, C., REDONDO, M.A., ORTEGA, M., VERDEJO, F. (2005) Collaborative environments for the learning of design: A model and a case study in Domotics. Computers \& Education. An International Journal, Pergamon.. En prensa.

BRAVO, J., ORTEGA, M., BRAVO, C., REDONDO, M.A. (2003) Planning: An intermediate solution to the problems in Design. en J.M. CUEVA, B. MARTÍN, L. JOYANES, J.E. LABRA, M. P. PAULE (eds.) Web Engineering. Proceedings of 3rd International Conference of Web Engineering. .LNCS 2722, 
Springer Verlag 99-107.

CETIS, (2001) Centre for Educational Technology Interoperability Standards. en http://www.cetis.ac.uk.

EduTools, (2004) http://www.edutools.info. Consultada el 20 de Octubre de 2004.

IEEE Learning Technology Standards Committee, (2001) Draft Standard for Learning Technology Systems Architecture LTSA. Draft 9., Disponible en http://ltsc.ieee.org/wg1/materials.html

IRS, (2004) E-learning Glossary, en

http://www.irs.gov/opportunities/procurement/article/0,,id=128685,00.html. Consultada el 20 de Octubre de 2004.

ORTEGA, M., (2000) Computers and Education: The near future. Plenary Lecture. en Computers and Education in the 21st Century. Selected papers from the Spanish Congress on Computers in Education (ConieD ’99), Kluwer Academic Publishers; 3-16.

ORTEGA, M. (2004) Sistemas de e-Learning y Colaboración: Guías para el desarrollo de Sistemas Colaborativos en web., Actas del “Web Development Workshop 2004”. F. J García Peñalvo y María de Navelonga Moreno (eds.), 49-59. Universidad de Salamanca.

ORTEGA, M., SÁNCHEZ-VILLALÓN, P.P.,(2005) AWLA: A Writing e-Learning Appliance, en Z. MA (ed.), Web-Based Intelligent e-Learning Systems: Technologies and Applications. En prensa.

REDONDO, M.A., BRAVO, C. ORTEGA, M., VERDEJO, F.(2002), PlanEdit: An adaptive tool for design learning by problem solving en P. DE BRA, P. BRUSILOVSKY, R. CONEJO (eds.) “Adaptative Hypermedia and Adaptative Web-Based Systems”. Proceedings of 2nd International Conference on Adaptative Hypermedia and Adaptative Web Systems. LNCS 2347; Springer Verlag, 560-563

REDONDO, M.A., BRAVO, C., BRAVO, J., ORTEGA, M. (2003a) Organizing activities of problem based collaborative learning with the DomoSim-TPC system. en M. LLAMAS, M.J. FERNÁNDEZ Y L.E. ANIDO (eds.), "Computers and Education: Towards a Lifelong Learning Society" Invited and selected papers from the VI Iberoamerican Congress on Computers in Education (IE'2002) and IV International Symposium on Computers in Education SIIE'2002, Kluwer Academic Publishers, 37-49.

REDONDO, M.A., BRAVO, C., BRAVO, J., ORTEGA, M. (2003b) Applying Fuzzy Logic to Analyze Collaborative Learning Experiences in an e-Learning Environment.USDLA Journal. (United States Distance Learning Association).17.2, 19-28.

PAREDES, M.,, SÁNCHEZ-VILLALÓN, P. P., ORTEGA, M., (2003) VELÁZQUEZ-ITURBIDE, J.A, (2003) A Ubiquitous Learning Model: e-Club, en M. LLAMAS, M.J. FERNÁNDEZ Y L.E. ANIDO (eds.), Computers and Education: Towards a Lifelong Learning Society. Invited and selected papers from the VI Iberoamerican Congress on Computers in Education (IE'2002) and IV International Symposium on Computers in Education SIIE’2002. Kluwer Academic Publishers 263-274.

SÁNCHEZ-VILLALÓN, P.P., ORTEGA, M. (2004) Writing on the Web: a Web appliance in a Ubiquitous e-Learning Environment, M. SINGHAL (ed.) The Reading Matrix: an International On-line Journal, 15-26. http://www.readingmatrix.com/onlineconference/ proceedings2004.html.

THORNE, S., SHUBERT, C., MERRIMAN, J. (2002). OKI architecture overview. OKI Project Document.en http://web.mit.edu/oki/learn/papers.html.

WEISER, M. (1991) The computer for the 21 ${ }^{\text {st }}$ Century, Scientific American, September, 94-104.

\section{Notas:}

[1] http://www.webct.com. 
[2] http://www.atutor.ca, http://moodle.org, http://www.claroline.net, http://dotlrn.org/

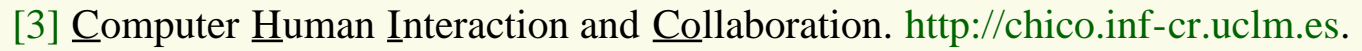

[4] $\underline{\text { A }}$ Writing eLearning Appliance. http://chico.inf-cr.uclm.es/ppsv/awla.html

(C) Ediciones Universidad de Salamanca

Webmasters del volumen actual: Francisco Ignacio Revuelta Domínguez,

Lourdes Pérez Sánchez

Correos electrónicos por orden: fird@usal.es

o bien lopesan@usal.es 